\title{
Heavy quark dynamics in the QGP: Towards a solution of the $R_{A A}$ and $v_{2}$ puzzle
}

F. Scardina ${ }^{1,2}$, S. K. Das ${ }^{2}$, S. Plumari ${ }^{1,2}$ and V. Greco ${ }^{1,2}$

${ }^{1}$ Department of Physics and Astronomy, University of Catania, Via S. Sofia 64, I-95123 Catania, Italy

${ }^{2}$ INFN-Laboratori Nazionali del Sud, Via S. Sofia 62, I-95123 Catania, Italy

\begin{abstract}
The two key observables related to heavy quarks that have been measured in experiments are the nuclear suppression factor $R_{A A}$ and the elliptic flow $v_{2}$. The simultaneous reproduction of these two observables is a puzzle which have challenged all the existing models. We discuss two ingredients responsible for addressing a large part of such a puzzle: the temperature dependence of the energy loss and the full solution of the Boltzmann collision integral for the scattering between the heavy quarks and the particle of the bulk.
\end{abstract}

\section{Introduction}

One of the primary aims of the ongoing nuclear collisions at Relativistic Heavy Ion Collider (RHIC) and Large Hadron Collider (LHC) energies is to create a Quark Gluon Plasma (QGP). Heavy Quarks (HQ), charm and bottom, created in ultra-Relativistic Heavy Ion Collisions (uRHIC) represents ideal probes to study the QGP $[1,2]$. An essential feature in analyzing Heavy quarks motion in a QGP is that their mass is much larger than the typical momentum exchanged with the plasma particles entailing that many soft scatterings are necessary to change significantly the momentum and the 
trajectory of the heavy quarks. Therefore the propagation of heavy quarks has been usually treated as a Brownian motion that is described by means of the Fokker-Planck (FP) equation.

The two key observables related to HQ that have been measured in experiments are the nuclear suppression factor $R_{A A}$ and the elliptic flow $v_{2}$ [3-5]. Several theoretical efforts have been made to study the $R_{A A}$ and the $v_{2}$ measured in experiments within the Fokker Planck (FP) approach $[6-9,11,12]$ and the relativistic Boltzmann approach (BM) [13-16]. However all the approaches show some difficulties to describe both the nuclear modification factor and the elliptic flow simultaneously.

To study the time evolution of $R_{A A}$ and $v_{2}$ we have solved the Fokker Planck (FP) equation stochastically in terms of the Langevin equation, for detail we refer to our previous work [11]. The evolution of the bulk is provided by a $3+1 \mathrm{D}$ relativistic transport code tuned at fixed $\eta / s$ [18] which is able to reproduce the same results of hydrodynamical simulations. We have carried out simulations of $A u+A u$ collisions at $\sqrt{s}=200 \mathrm{AGeV}$ for the minimum bias. The HQ distribution in momentum space is in accordance with the one in proton-proton rescaled by the number of binary collisions. We have used three different modelings to calculate the drag coefficient. The diffusion coefficient is instead calculated in accordance with the Einstein relation $D=\Gamma E T$.

In the first modeling we have evaluated the drag coefficient from (pQCD) and we have considered elastic interaction among $\mathrm{HQ}$ and the bulk (light quarks and gluons). The scattering matrix related to these processes $\mathcal{M}_{g H Q}$, $\mathcal{M}_{q H Q}$ and $\mathcal{M}_{\bar{q} H Q}$ in leading order are the well known Combridge matrix. The infrared singularity in the $t$-channel is regularized introducing a Debye screening mass $m_{D}=\sqrt{4 \pi \alpha_{s}} T$ with a running coupling.

We have also considered another modeling in which the drag coefficient is evaluated considering a bulk consisting of particles with a T-dependent quasi-particle masses, $m_{q}=1 / 3 g^{2} T^{2}, m_{g}=3 / 4 g^{2} T^{2}$. This model is able to reproduce the thermodynamics of lattice QCD [20] (see also [21]) by fitting the coupling $g(T)$. Such a fit leads to the following coupling [20]:

$$
g^{2}(T)=\frac{48 \pi^{2}}{\left[\left(11 N_{c}-2 N_{f}\right) \ln \left[\lambda\left(\frac{T}{T_{c}}-\frac{T_{s}}{T_{c}}\right)\right]^{2}\right.}
$$

where $\lambda=2.6$ and $T / T_{s}=0.57$.

Finally we have considered a model in which the light quarks and gluons are massless but the coupling is from the QPM as indicated in Eq. 1. This last case is indicated in the figures as $\left(\alpha_{Q P M}(T), m_{q}=m_{g}=0\right)$ and has 

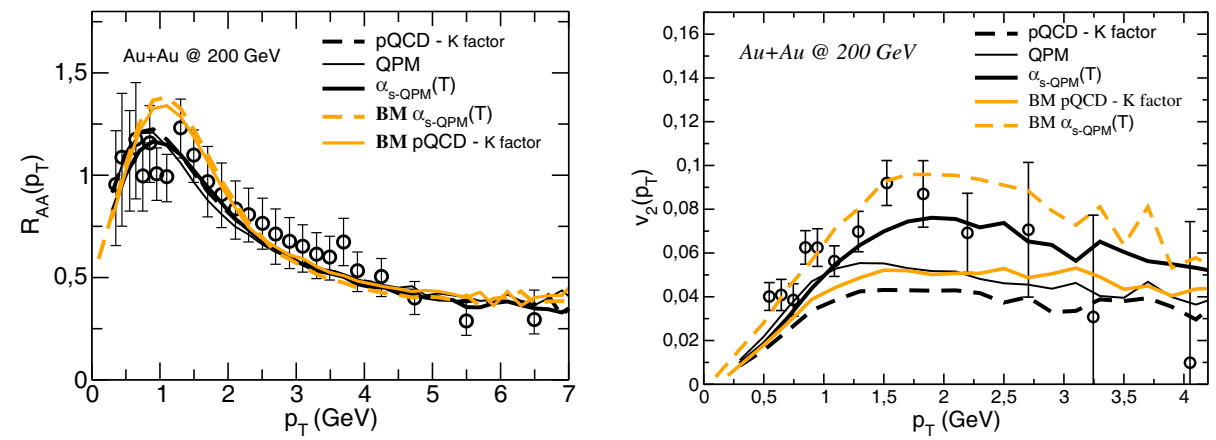

Figure 1: Comparison of the experimental data for the $R_{A A}$ (left) and $v_{2}$ (right)at $(200 \mathrm{AGeV})$ with the results we get using the different models. See text.

to be considered as an expedient to have a drag which increases when the temperature decreases.

For all the three cases considered the interaction has been rescaled to reproduce the $R_{A A}$ observed in experiments. We have performed simulations of HQ propagation with the Langevin dynamics for the three different models presented above. The Langevin equation gives as output the momentum distributions of $\mathrm{HQ}$ at the quark-Hadron transition temperature $T_{c}$. The momenta distributions are convoluted with the Peterson fragmentation functions of the heavy quark to get the momentum distribution of $\mathrm{D}$ and $\mathrm{B}$ mesons.

In Fig. 1 (left) the nuclear modification factor $R_{A A}$ of the $\mathrm{D}$ and $\mathrm{B}$ mesons is shown as a function of $p_{T}$ for RHIC (200AGeV). Instead in Fig. 1 (right) the elliptic flow $\left(v_{2}\right)$ at the same energy as a function of $p_{T}$ is depicted. We observe that the larger is the interaction in the region of low temperature the larger is the elliptic flow. The same conclusions has been discussed also in the light flavor sector as shown in Ref. [22]. The reason of such a strong dependence of the elliptic flow on the temperature dependence of the drag coefficient is due to the fact that the elliptic flow is generated in the final stage of the evolution of the fireball when the temperature is lower. This is shown in the Fig. 2 where the $R_{A A}$ (left) and $v_{2}$ (right) are depicted at different times. We observe that the $R_{A A}$ is generated in the early stage of the QGP when the temperature is larger and is not sensitive to the final stage of the evolution, while the elliptic flow is generated later. This results refer to the pQCD case, however the behavior is similar also for the other models.

We have also used the transport approach to study the propagation of Heavy quarks. The Boltzmann equation for the HQ distribution function is 

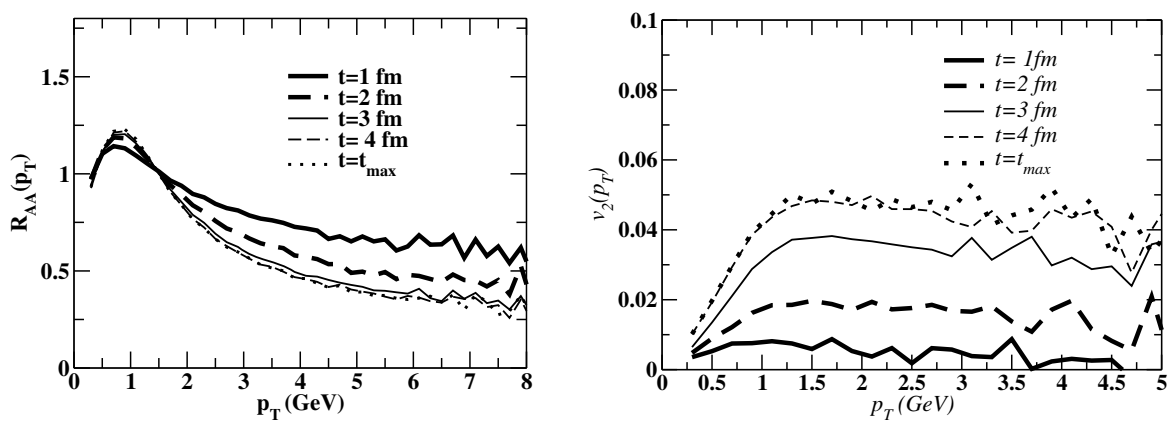

Figure 2: $R_{A A}$ (left) and $v_{2}$ (right) evaluated at different time at RHIC energies $(200 \mathrm{AGeV})$.

indicated here

$$
p^{\mu} \partial_{\mu} f_{Q}(x, p)=\mathcal{C}\left[f_{Q}\right](x, p)
$$

where $\mathcal{C}\left[f_{Q}\right](x, p)$ is the relativistic Boltzmann-like collision integral which is solved by means of a stochastic algorithm. We use the Boltzmann equation to describe the propagation of the heavy quark as well as the evolution of the bulk.

The comparison between LV and BM approach has been thoroughly studied in these references $[15,16]$ where it is shown that for charm quark the results that one gets using the Fokker-Planck approach deviate significantly from those obtained using the Boltzmann approach and such a deviation significantly depends on the the values of the Debye screening mass, whereas for bottom quarks the FP is a very good approximation. We considered in references $[15,16]$ three values of $m_{D}: 0.4 \mathrm{GeV}, 0.83 \mathrm{GeV}$ and $1.6 \mathrm{GeV}$. Here we have not considered a fixed value of the Debye screening mass but a value which depends on the temperature according to $m_{D}=g T$. In Fig. 1 the comparison for the $R_{A A}$ (left) and $v_{2}$ (right) at RHIC between the BM (light gray lines) and the FP (black lines) are shown. We found that using the $\mathrm{BM}$ for the same values of the $R_{A A}$ we get larger values for $v_{2}$ with respect to those obtained using the FP.

Our results show a non-negligible impact of the approximation in the collision integral involved in the Fokker Plack equation on the relation between $R_{A A}$ and $v_{2}$. We found that for the same $R_{A A}$ we get a larger $v_{2}$ using the $\mathrm{BM}$ with respect to the $v_{2}$ we get with the FP.

To summarize our results, regarding the different values of elliptic flow that can be obtained using the different models of energy loss, we have introduced in Fig. 3 a new plot in which $R_{A A}$ vs $v_{2}$ at a given momentum $\left(p_{T}=1.3\right.$ $\mathrm{GeV}$ ) is shown. 


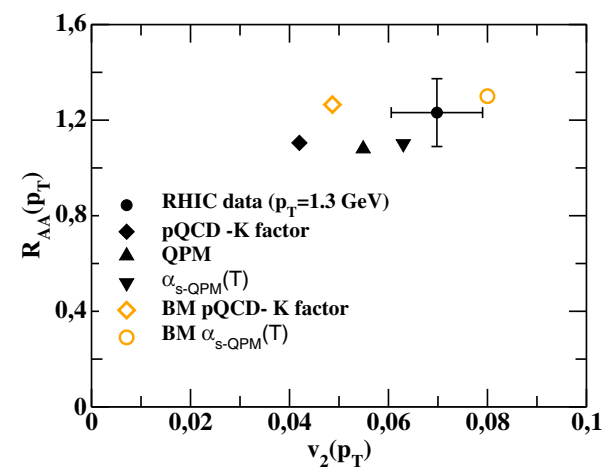

Figure 3: $R_{A A}$ vs $v_{2}$ obtained with the FP for the three different $\mathrm{T}$ dependences of the drag coefficient with the experimental data at RHIC energy at $p_{T}=1.3 \mathrm{GeV}$. The open light gray simbols indicates the results obtained using the BM approach.

This plot clearly shows how the building up of the $v_{2}$ can differ up to a factor 3 for the same $R_{A A}$ depending on the temperature dependence of the energy loss and on the approach used to describe the HQ propagation. Other two ingredients that can have an impact on the relation between $R_{A A}$ and $v_{2}$ are the hadronization via coalescence that we are going to include in our description and the role of the hadronic phase that we have studied in [23]. We have found in such a study that the hadronic medium further enhance the $v_{2}$ by around $20 \%$ without affecting the nuclear modification factor.

\section{Acknowledgments}

The authors acknowledge the support by the ERC StG under the QGPDyn Grant n. 259684.

\section{References}

[1] Svetitsky B 1988 Phys. Rev. D 37, 2484

[2] R.Rapp and H van Hees, R. C. Hwa, X. N. Wang (Ed.) Quark Gluon Plasma 4, 2010, World Scientific, 111

[3] B. I. Abeleb (STAR Collaboration), Phys. Rev. Lett. 98, 192301 (2007).

[4] A. Adareet al. (PHENIX Collaboration) A. Adare et al. (PHENIX Collaboration), Phys. Rev. C 84 , 044905 (2011)

[5] B. Abelev et al.,(ALICE Collaboration) JHEP 1209 (2012) 112

[6] Hees H van, Greco V and Rapp R 2006 Phys. Rev. C 73, 034913; Hees H van, Mannearelli M, Greco V and Rapp R 2008 Phys. Rev. Lett. 100 192301 
[7] Das S K, Alam J, Mohanty 2009 Phys. Rev. C 80 054916;

[8] Alberico W M et al. 2011 Eur. Phys. J. C 71 1666;

[9] S. Cao, G. Y. Qin and S. A. Bass, Phys. Rev. C 88 (2013) 4, 044907

[10] M. He, R. J. Fries and R. Rapp, Phys. Rev. Lett. 110, 112301 (2013)

[11] S. K. Das, F. Scardina, S. Plumari and V. Greco, Phys Lett. B747 (2015) 260-264

[12] F. Scardina,S. K. Das, S. Plumari,J. I. Bellone and V. Greco, arXiv:1509.01551

[13] Gossiaux P B, Aichelin J 2008 Phys. Rev. C 78014904

[14] Uphoff J, Fochler O, Xu Z and Greiner C 2011 Phys. Rev. C 84024908

[15] S. K. Das, F. Scardina, S. Plumari and V. Greco,Phys. Rev. C 90 044901 (2014)

[16] F. Scardina,S. K. Das, S. Plumari and V. Greco, J.Phys.Conf.Ser. 535 (2014) 012019; F. Scardina,S. K. Das, S. Plumari and V. Greco, J.Phys.Conf.Ser. 636 (2015) 012017

[17] Younus M, Coleman-Smith C E,Bass S A and Srivastava D K, Phys.Rev. C 91 (2015) 2, 024912

[18] S. Plumari,A. Puglisi, F. Scardina and V. Greco, Phys. Rev. C 86 (2012) 054902

[19] V. Greco, C. M. Ko and P. Levai, Phys. Rev. Lett. 90 (2003) 202302;

V. Greco, C. M. Ko and P. Levai, Phys. Rev. C 68 (2003) 034904

[20] S. Plumari, W. M. Alberico, V. Greco and C. Ratti, Phys. Rev. D, 84, 094004 (2011)

[21] H. Berrehrah, E. Bratkovskaya, W. Cassing, P.B. Gossiaux, J. Aichelin, and M. Bleicher, Phys.Rev. C 89, 054901 (2014),

[22] F. Scardina, M. Di Toro and V. Greco, Phys. Rev. C 82 (2010) 054901.

[23] S. K. Das, F. Scardina, S. Plumari and V.Greco, arXiv:1509:06307 